\title{
Clotho et la programmation de la sénescence
}

«Mourir, cela n'est rien, mais vieillir... $\mathrm{Ah}$ ! vieillir...» La sénescence inéluctable - ride véloce, démarche hésitante, artériosclérose, ostéoporose, cataracte et démence - fait cortège au grand âge. Chez les humains surtout, car d'autres mammifères (les souris, par exemple, dont la longévité est d'environ trois ans) sont généralement préservés de ces signes de vieillissement. C'est pourtant en étudiant des souris transgéniques rendues hypertendues par surexpression d'un gène codant pour un échangeur sodium-proton qu'une équipe japonaise [1] vient de faire une découverte qui pourrait bien concerner ce programme de la sénescence [2]. Certes, d'autres gènes, comme les gènes de la réparation de l'ADN $(\mathrm{m} / \mathrm{s}$ $n^{\circ}$, vol. 12, p. 403) [3] peuvent, lorsqu'ils sont mutés, provoquer vieillissement et cancers parce qu'ils s'attaquent à la vie même de la cellule, mais ils ne participent pas vraiment au programme de la sénescence [4]. L'équipe japonaise n'avait rien laissé au hasard: au lieu de se désintéresser des souris transgéniques qui n'avaient pas développé d'hypertension, Makoto Kuro-o et son groupe les croisèrent avec d'autres souris transgéniques non hypertendues afin de rechercher d'éventuelles homozygotes ou l'apparition de phénotypes nouveaux par mutation insertionnelle. Effectivement, ils observèrent une souris à vie brève et développèrent toute une lignée pour obtenir des homozygotes: vieillies avant l'âge, chenues et rabougries, elles offraient tous les signes physiques de sénilité que l'on observe chez les humains du quatrième âge: cyphose, hypokinésie, trouble de l'équilibre, atrophie des organes génitaux (avec absence de libido), calcifications ectopiques (dans les parois artérielles et divers organes), diminution de la masse osseuse, atrophie cutanée, emphysème et hypoplasie hypophysaire. Le tableau clinique était si homogène et si exceptionnel, avec une durée de vie si brève (n'excédant pas 8 à 9 semaines) que l'équipe nippone abandonna illico l'étude de l'hypertension pour traquer ce gène dont les mutations à l'état homozygote réalisaient un modèle animal caractérisé $\mathrm{du}$ vieillissement humain. D'abord localisé grâce au repérage du transgène en hybridation in situ, il fut ensuite cloné et baptisé Klotho (kl) du nom d'une des trois Parques qui tiennent dans leurs mains le fil du destin des hommes: Clotho est la fileuse, Lachesis l'enroule autour du fuseau et Atropos coupe le fil. Ce gène code pour une protéine de surface cellulaire comportant une seule hélice transmembranaire et deux domaines extracellulaires (KL1 et KL2) ayant chacun une similitude de séquence avec les $\beta$-glucosidases des plantes et des bactéries ainsi qu'avec la lactase glycosyl-céramidase des mammifères. Les souris atteintes avaient, à l'état homozygote, une délétion dans la région 5' du gène et la réintroduction du gène $k l$ normal dans des embryons de souris homozygotes faisait disparaître l'ensemble des signes de progéria et recouvrer une fertilité et une durée de vie normales. Le gène humain correspondant est localisé en $13 q 12$ et code pour une protéine ayant $86 \%$ d'identité avec la protéine KL murine. S'il s'agit vraiment d'un gène impliqué dans la longévité et la sénescence, il faut constater que Klotho fait très fort! Le décès très précoce des souris (correspondant à un âge humain de six ans), leur petite taille, l'hypotrophie de l'hypophyse font regretter de ne l'avoir pas prénommé Atropos! Il évoque un des rares syndromes progéroïdes transmis en récessivité, le syndrome de Wiedemann-Rautenstrauch qui est le plus souvent létal dans l'enfance [5]. La protéine déduite laisse supposer que Klotho serait impliqué dans le métabolisme des sphingolipides $\left(m / s n^{\circ} 3\right.$, vol. 9, p. 339) agissant sur les gangliosides et les glycolipides de membrane pour libérer du céramide. Voici enfin ouverte cette "piste du céramide» pour la sénescence et l'apoptose $(\mathrm{m} / \mathrm{s}$ $n^{\circ} 6-7$, vol. 9, p. 813) sur laquelle nous insistions déjà en $1996\left(\mathrm{~m} / \mathrm{s} n^{\circ} 5\right.$, vol. 12, p. 658).

S.G.

\footnotetext{
1. Kuro-o M, Matsumura Y, Aizawa H, Kawaguchi $\mathrm{KH}$, Suga T, et al. Mutation of the mouse klotho gene leads to a syndrome resembling ageing. Nature 1997; 390: 45-51.

2. Dreyfus JC, Kahn A. Any theory of ageing must be universal. Trends Biochem Sci 1977; 2: 205-6.

3. Kahn A. Réparation, cancer et sénescence: le gène du syndrome de Werner. Med Sci 1996; 12: 802-4.

4. Sarasin A. Les gènes humains de la réparation de l'ADN. Med Sci 1994; 10 : 43-54.

5. Bitoun P, Lachassine E, Sellier N, Sauvion S, Gaudelus J. The Wiedemann-Rautenstrauch neonatal progeroid syndrome: a case report and review of the literature. Clin Dysmorph 1995; 4 : $239-45$
}

\section{$3^{\text {es }}$ JOURNÉES D'ACTUALITÉS EN PATHOLOGIE OSSEUSE L'HYPER-RESORPTION OSSEUSE ET SES TRAITEMENTS} 3-4 avril 1998 - ANGERS - Centre de Congrès

Organisation : $\quad$ Service de Rhumatologie, CHU d'Angers, LHEA Laboratoire d'Histologie - Embryologie, CHU et Faculté de Médecine d'Angers

Sous les auspices de : GRIO (Groupe de Recherche et information sur l'ostéoporose), IFFSD (International Federation for Skeletal Diseases), Société Française de Rhumatologie, IFM (Institut Français du Myélome), SRO (Société de Rhumatologie de I'Ouest)

Secrétariat : Mme D. Dumont, LHEA Laboratoire d'Histologie-Embryologie. Faculté de Médecine - 49045 Angers Cedex, France. Tél. : 0241735864 - Fax : 0241735888 